\title{
Endurer la surprise
}

\section{Emmanuel de Saint Aubert}

\section{OpenEdition}

\section{Journals}

Édition électronique

URL : http://journals.openedition.org/alter/428

DOI : 10.4000/alter.428

ISSN : 2558-7927

\section{Éditeur :}

Association ALTER, Archives Husserl (CNRS-UMR 8547)

\section{Édition imprimée}

Date de publication : 1 décembre 2016

Pagination : 123-142

ISBN : 978-2-9550449-2-6

ISSN : 1249-8947

Référence électronique

Emmanuel de Saint Aubert, «Endurer la surprise », Alter [En ligne], 24 | 2016, mis en ligne le 01 décembre 2017, consulté le 21 avril 2019. URL : http://journals.openedition.org/alter/428 ; DOI : $10.4000 /$ alter.428 


\title{
ENDURER LA SURPRISE
}

\author{
Emmanuel de Saint Aubert
}

\section{Active passivité}

La surprise, en tant que telle, implique un "dehors», un événement dans notre relation au monde : elle n'est pas purement immanente à notre vie psychique. Il est impossible de se faire à soi-même une véritable surprise, il est bien difficile de simuler mentalement un effet de surprise. Plus transversal qu'un simple sentiment, le phénomène de la surprise peut solliciter l'ensemble de notre être, à commencer par notre institution corporelle la plus élémentaire. La surprise nous prend de vitesse et ne nous laisse pas sur place : elle a un impact sur la spatio-temporalité de notre chair. Ce n'est pas là une question de position, mais de situation. La surprise nous déstabilise, de manière imperceptible ou de manière radicale; elle peut faire vibrer voire ébranler les fondements de notre être-au-monde : faire vaciller notre équilibre, depuis les dimensions posturales du schéma corporel $^{1}$, jusqu'aux dimensions les plus profondes d'une identité personnelle et relationnelle portée par l'image inconsciente du corps². $\hat{A}$ travers et au-delà de ses aspects psychologiques, la surprise a en somme un caractère existentiel.

Si la surprise nous dépasse et nous déplace, si elle nous devance depuis un dehors de nous-mêmes et peut traverser tout notre être, si elle est ainsi en quelque sorte ontologiquement transcendante et

\footnotetext{
${ }^{1}$ Cette question de l'équilibre, comme adaptation aux perpétuelles déstabilisations que notre environnement nous fait vivre, habite d'ailleurs les fondements mêmes de la notion de schéma corporel, dès ses linéaments chez les neurologues Pierre Bonnier (l'aschématie, la question du vertige) et Henry Head (le schéma postural).

2 Cf. E. de Saint Aubert, Être et chair I, Paris, Vrin, 2013, section A.
} 
anthropologiquement transversale, se pose néanmoins la question délicate de la nature de notre passivité à son égard. Un ensemble de questions, à vrai dire. Si nous sommes surpris, n'est-ce pas parce que l'événement impliqué s'écarte de ce que nous attendions? Ce qui suppose une attente, voire une anticipation, et une capacité à percevoir cet écart. Et pour que la surprise nous déstabilise, voire nous fasse sortir de nous-mêmes, encore faut-il être disposé à être ainsi dépassé et déplacé, encore faut-il être ouvert à la surprise, voire $\mathrm{s}^{\prime}$ attendre à être surpris. Pour le dire simplement, il ne va pas de soi d'être surpris, et d'endurer la surprise. Il y a des conditions à cette passivité, et celle-ci est en réalité lourde d'une sourde activité.

Nous allons tenter de déplier cette complexion de notre active passivité dans la surprise. Commençons par une première distinction, qui sera affinée par la suite. La surprise se compose d'une double « prise » : être pris, certes, mais aussi saisir que l'on est pris, ce qui est déjà commencer à (re-)prendre. (1) Être pris. On dit ainsi "surprendre » quelqu'un: il est pris en flagrant délit, saisi la main dans le sac... Mais encore faut-il se faire prendre, se laisser saisir. Or, l'être humain peut montrer une étonnante habileté, sinon une véritable tendance, à résister voire échapper à la saisie de la surprise. J'y reviendrai. (2) Reprendre. Être surpris suppose d'avoir un minimum perçu et reçu la nouveauté comme telle : d'avoir perçu un écart entre ce que nous attendions (voire anticipions) et ce que nous percevons. D'une certaine manière, nous surprenons la surprise. Mais encore faut-il être en mesure de percevoir, avec le minimum de tension et $\mathrm{d}$ 'attention que cela suppose, et encore faut-il que l'écart ne soit pas trop grand, au risque de n'être même plus un écart.

Etre en mesure de percevoir (la surprise) requiert d'être un tant soit peu en prise sur le monde (pour être sur-pris). Merleau-Ponty pensait qu'on l'est toujours, même dans le sommeil - mais avec bien des différences de degré tout de même, de manière plus ou moins forte, et parfois plus ou moins pathologique. Le même MerleauPonty, qui s'intéressait à la psychologie des "méthodes projectives », s'est ainsi penché sur les mécanismes de la psychorigidité, qui se traduit par une faible perception des changements, ou un retard de celle-ci ${ }^{3}$. Indépendamment de toute psychorigidité, on peut être occupé, préoccupé, à tel point que la surprise est soit quasi impossible soit trop brutale. Ainsi lorsque quelqu'un est si concentré et absorbé par une tâche qu'il n'est pas atteint, ou encore si profondément endormi que l'on n'arrive pas à le réveiller, à le surprendre dans son

${ }^{3}$ Cf. E. de Saint Aubert, Être et chair I, op. cit., chap. VII. 
sommeil. Ou bien la surprise provoque un tel sursaut, une telle déstabilisation, que le phénomène $n^{\prime}$ est pas ou pas encore une surprise: on n'est pas véritablement pris, saisi par quelque chose de nouveau, mais simplement dans le vide, perdu et non pas pris, et il faut $s^{\prime} y$ re-prendre... $s^{\prime} y$ reprendre à deux fois pour être surpris.

Pour qu'il y ait surprise, ne faut-il pas que le vivant, l'animal que nous sommes, participe un tant soit peu au déplacement, à la déstabilisation? Mais encore faut-il qu'il ait la marge de le faire. Si l'écart est trop grand... Ainsi d'un ami qui a eu un grave accident de moto, à un moment où, en pleine course, il faisait l'erreur de regarder en arrière celui qui le suivait. Il a vécu alors un événement parfaitement contraire à son anticipation, qui l'a conduit à quitter la route et retomber trente mètres plus loin, sans avoir jamais perdu connaissance. Y a-t-il eu surprise ? Il a été dépassé, déplacé, ouvert - au sens le plus propre, et jusque dans ses os. Mais y a-t-il encore surprise quand on est ainsi réduit à une pure passivité, que l'on est perdu, sans repère, dans un vertige généralisé, si bien que l'on n'a rien à dire, à en dire, sinon (et encore, au bout d'un moment) « je ne sais pas ce qui m'est arrivé ». La dés-orientation est totale, il n'y a pas d'écart, et il va falloir du temps pour petit à petit assembler les pièces d'un puzzle, faire du lien, sortir du vertige, construire des écarts et pas seulement subir l'abîme d'une totale et radicale séparation. On pourrait ainsi multiplier les exemples, bien connus des urgentistes ou des services de police, appelés à accueillir des personnes si traumatisées par un accident ou une agression qu'elles sont incapables de décliner leur identité pendant plusieurs heures. Ces exemples illustrent autant de phénomènes de vertige, $\mathrm{d}$ 'aschématie plus ou moins généralisée, du schéma postural jusqu'à l'identité consciente. La déstabilisation est allée trop loin, et cette radicale prise sans possible reprise ne constitue pas une surprise.

En somme, lorsque nous maintenons les horizons du monde audelà d'un rempart de repères rigides, lorsque nous sommes absorbés par une mécanique de déterminations qui résorbe momentanément ou durablement toute indétermination, nous ne pouvons pas être surpris (sauf à basculer dans le vide, dans l'hébétement d'un état de vertige, ce qui est encore échapper à la surprise). Et si nous n'avons pas ou plus de repères, si nous sommes déjà en état de vertige, sans monde, nous ne pouvons pas non plus être surpris. Pour pouvoir être surpris, il nous faut être ouverts à l'indétermination tout en nous appuyant sur des déterminations. Ce qui est une façon schématique de dire qu'il nous faut être au monde. Être au monde dans une attitude perceptive, qui n'isole pas la figure du fond dont elle émerge, qui voit 
en profondeur, saisit le visible tout en s'ouvrant à l'invisible qu'il porte et qui le porte, s'oriente sur des repères tout en gardant le cap sur l'horizon. Etre au monde dans la foi perceptive, la foi consistant justement à s'ouvrir à l'indétermination tout en s'appuyant sur des déterminations - à passer outre à l'insuffisance de ce que nous savons et ressentons, tout en nous appuyant sur ce que nous savons et ressentons déjà.

\section{S'adapter}

Avant de développer ce cadre perceptif de notre ouverture à la surprise, analysons plus avant la doublure passive-active de l'endurance de la surprise. Je distinguerai maintenant trois phases, en réalité étroitement intriquées, en m'inspirant notamment d'une clinique qui m'est chère, celle de l'éducation spécialisée. (1) La première phase, en deçà d'un véritable rapport à l'écart ou à la nouveauté, consiste à consentir à une forme de "dé-prise »: un ébranlement de certains repères, une modification des représentations, voire une dimension de perte et de séparation. Certains n'y parviennent pas, ou peu, s'accrochant désespérément à ce qu'ils savent, maîtrisent - aux quelques repères qu'ils ont peut-être conquis difficilement et douloureusement pour tenir leur tête hors de l'eau, au-dessus du gouffre du non-sens. La psychorigidité est ici conduite de sauvegarde. (2) La déstabilisation de la surprise ne s'arrête pas à ce lâcher-prise, et invite ensuite à endurer l'instabilité pour elle-même, une indétermination plus ou moins relative et momentanée. Certains parviennent à lâcher prise, mais tombent ensuite en état de vertige, avec la part d'angoisse qui l'accompagne : l'indétermination est pour eux abîme, la profondeur sol qui se dérobe, le mystère non-sens. Capables de lâcher, ils ne parviennent pas à plonger. Il n'y aura pas pour eux de surprise, mais une stupeur, faite $\mathrm{d}^{\prime} u n$ blocage cognitif éventuellement accompagné d'éclatement perceptif et comportemental, un immobilisme intellectuel éventuellement accompagné d'agitation corporelle désordonnée, de mouvements qui ne font pas geste. (3) Consentir à la dé-détermination et endurer l'indétermination ne suffisent pas. Encore faut-il endurer la nouveauté jusqu'à s'ouvrir à son éventuelle positivité. Consentir à se laisser travailler par elle, à se laisser prendre tout en la prenant, tout en l'apprenant; se laisser comprendre tout en la comprenant; l'accueillir en vue de l'intégrer un minimum, quitte à la filtrer et à finalement la rejeter. Après avoir lâché, et plongé, il faut tenter de voler. C'est ici sans doute le processus le plus complexe, et 
le plus riche d'active passivité, qui porte à son sommet l'épreuve que constitue la surprise. Celui qui vole s'abandonne à la portance de l'air dans un minimum de foi dans le fait que cette apparente indétermination n'est pas que du vide, et qu'il peut s'appuyer sur une certaine consistance et cohérence du mode d'être de cet élément (ce faisant, cet acte de foi, en tant que tel, consiste déjà à s'appuyer dessus). Un mode d'être qu'il doit apprivoiser dans un effort d'adaptation.

Commence ainsi, au-delà de la surprise, mais déjà initié en son sein même, tout un processus de re-prise. Ce processus n'est pas une pure activité à sens unique, parfaitement postérieure à la passivité de la dé-prise, mais une dynamique passive-active où l'intégration de la nouveauté demande de se laisser transformer (accommodation) et de transformer (assimilation) : incorporer la nouveauté, dans un corps-àcorps où s'opère une transfiguration - se laisser configurer par la nouveauté, la figurer et la digérer, pour qu'elle commence à faire corps avec nous. Accommodation, assimilation, mais aussi, et plus souvent qu'on pourrait le croire, une part vitale de rejet. Car l'incorporation ne consiste pas à tout introjecter, au risque de résorber la nouveauté sans l'intégrer, de l'annexer sans se laisser véritablement surprendre, et sans sur-prendre la surprise. Une accommodation et/ou une assimilation totales ne signent pas forcément la réussite de l'adaptation. Plusieurs cas de figure seraient ici à envisager. On peut ne rien (oser) rejeter par peur d'être rejeté ; ou se laisser totalement configurer dans une posture dépressive qui dit passivement oui à tout, jouant ainsi les stupides pour ne pas endurer la surprise; ou encore tout assimiler pour, en réalité, ne rien avoir à assimiler, et finalement tout vomir et rejeter, dans un masochisme secrètement destructeur (de la nouveauté). Autant de torsions de l'incorporation qui déforment la surprise, ne parviennent pas à la vivre pleinement, voire la fuient tout en préservant les apparences de l'endurer.

Cette description nourrie d'observations cliniques croise des auteurs dont la conciliation n'a pourtant rien d'évident. Piaget, et son approche de l'intelligence comme adaptation, faite d'assimilation et $\mathrm{d}^{\prime}$ accommodation ; Freud, et plus largement l'approche psychanalytique de l'incorporation; Merleau-Ponty enfin, marqué par ceux-ci (et, s'agissant, de l'incorporation, par Melanie Klein), et développant pour son compte une phénoménologie de la chair " passive-active». Merleau-Ponty voit dans les va-et-vient ici suggérés (se laisser prendre / prendre en soi, s'abandonner / incorporer), et dans la relation circulaire et réciproque qu'ils dessinent, le double mouvement typique de la chair dans son commerce avec le monde, une relation faite 
de pénétration et d'enveloppement mutuels. Un double mouvement où s'exercent deux dynamiques fondamentales de notre animation : la foi, et le désir.

L'un des intérêts du riche phénomène de la surprise, décidément anthropologiquement transversal, est justement de convoquer et d'articuler ces dimensions trop facilement considérées comme hétérogènes : le corps, l'intelligence, la foi et le désir. Merleau-Ponty ne situe en aucun cas foi et désir du seul côté d'une affectivité dépourvue de dimension cognitive ; par ailleurs, il critique vigoureusement la conception piagétienne de l'intelligence comme se construisant dans une rupture avec la sensori-motricité en général et la vie perceptive en particulier. C'est justement dans la perception, à laquelle tant de ses réflexions sont consacrées, que Merleau-Ponty voit naître conjointement ce que d'autres nommeraient le cognitif et l'affectif : une ouverture tout à la fois confiante, désirante et intelligente à l'indétermination.

\section{Percevoir}

Revenons donc à la perception, pour mieux comprendre en quoi notre capacité à être surpris et à endurer la surprise engage une attitude perceptive - la surprise, en retour, contribuant à éveiller et creuser celle-ci. Fasciné par la façon dont la vue peut s'ouvrir à la profondeur et aux horizons, Merleau-Ponty envisage plus largement la perception comme capacité à s'ouvrir à et endurer l'inépuisable, lequel conjugue à la fois du visible, de l'invisible et de l'imprévisible : $\mathrm{s}^{\prime}$ ouvrir à et endurer cela même d'où peut surgir la surprise. Cette conception s'illustre notamment à travers deux directions de travail : une réflexion sur notre rapport aux «ultra-choses", emblématique de notre être-au-monde, et une analyse de la vision de l' "écart », dans laquelle Merleau-Ponty décèle un motif transversal de la vie perceptive.

a) La surprise des ultra-choses

Piaget voit l'intelligence se développer dans un rapport duel avec les choses, qu'elle constitue peu à peu en objets, par l'accès à la représentation puis à la réversibilité logique. L'objet est par définition et par construction « permanent » : je continue à croire à son existence en l'absence de données sensori-motrices immédiates, parce que je suis capable de suppléer à sa présentation perceptive par une représentation mentale. Le passage de la chose à l'objet repose ainsi sur 
la conquête d'une certaine accessibilité, par un gain de savoir, d'avoir et de pouvoir. Savoir que la chose perdure à son absence sensible, pouvoir se la donner à soi-même par la puissance de la représentation, le tout dans la motivation de la retrouver et de la posséder. Accessible et observable, l'objet est manipulable voire transformable, physiquement et mentalement. Le tout dessine comme horizon une progressive détermination de l'indétermination, dans un idéal d'observation et de maitrise, de circonscription physique et symbolique de l'être de la chose, d'épuisement de son sens. Un idéal de prise.

Se démarquant de la primauté accordée par Piaget aux objets ainsi conçus, Henri Wallon ${ }^{4}$ et à sa suite Merleau-Ponty invoquent l'existence $d^{\prime}$ " ultra-choses », infra-objets inaccessibles, non manipulables, qui échappent à cet idéal. Certaines réalités ou dimensions du réel résistent à nos procédés d'objectivation, en raison de nos limitations intellectuelles ou du fait de notre situation existentielle ; elles demeurent imparfaitement déterminées ou indéterminables, inépuisables ou imprévisibles - toujours aptes à nous surprendre. Loin de leur être indifférente, l'intelligence humaine est sollicitée, et même mobilisée par elles, malgré leur inaccessibilité. Malgré, mais aussi grâce à elle : si les ultra-choses sont loin de nous fournir une satisfaction pleine et immédiate, le fait qu'elles nous résistent, nous frustrent et soient adverses est susceptible d'être moteur, et même au plus haut point, de notre ouverture au monde.

L'analyse merleau-pontienne des ultra-choses vient enrichir sa phénoménologie de la perception la plus tardive, ainsi que les horizons anthropologiques et ontologiques de celle-ci. Notre rapport aux ultra-choses engage le corps et l'intelligence, l'imaginaire et le désir il engage la chair. Il illustre en particulier combien l'intelligence, contrairement à ce que pense Piaget, garde de bout en bout une dimension perceptive forte, qui n'est pas que le résidu de sa première phase (sensori-motrice), puisqu'elle porte aussi ses virtualités les plus abouties. L'intelligence poursuit et accentue ce qu'inaugurait déjà la vision, cette capacité à s'ouvrir à l'indétermination, à endurer l'inépuisable et anticiper l'invisible. En particulier, elle poursuit et accentue ce qu'inaugurait la perception de la profondeur. Ultra-chose qui n'est même pas chose, la profondeur enveloppe et porte toute chose, la met en tension avec ses côtés cachés et avec les autres choses, l'ouvre sur autre chose qu'elle-même.

${ }^{4}$ Cf. Les origines de la pensée chez l'enfant, Paris, PUF, 1945. 
L'intelligence n'est pas uniquement faite pour l'objet, fermé et achevé, pour un observable manipulable, mais aussi, et surtout, pour la surprise des ultra-choses, imprévisibles et inépuisables. Si bien qu'elle n'est pas ou ne devrait pas être d'abord motivée par la clôture du savoir, mais par l'indétermination des ultra-choses revisitées comme "mystères », dont la profondeur est comme l'inscription esthésiologique. L'intelligence, et plus largement la chair: l'intelligence entendue dans ses fondements les plus corporels et ses évolutions les plus subtiles, dans son lien irréductible avec le désir et la foi perceptive. Il y a en nous, depuis l'inachèvement constitutif de notre institution corporelle, une ouverture et même une appétence pour les horizons et la profondeur, pour l'invisible. C'est ce que MerleauPonty nomme notre "ouverture à l'être »- une disposition fondamentale à être surpris, à endurer la surprise de ces ultra-choses qui, en retour, éveillent et creusent notre perception.

b) L'épreuve de l'écart

Aux prises avec les ultra-choses, le sujet percevant est pourtant rarement seul face à elles. Du moins a-t-il besoin de s'appuyer sur des déterminations - sur des choses, sinon des objets - pour tolérer l'inaccessible et recevoir sa portance. Si Merleau-Ponty n'a pas suffisamment travaillé ce point, demeurant aveugle au pouvoir inhibant sinon destructeur des ultra-choses, et à notre ambivalence conséquente à leur égard, il y contribue néanmoins par son analyse de la perception comme épreuve d'un écart. Repartant de l'approche gestaltiste de la dualité de la figure et du fond, il souligne combien on ne perçoit jamais une chose isolément, mais toujours sur fond de... et dans un réseau de tensions qui à la fois relient et écartent la chose perçue et les autres choses. La perception est nativement mobilisée par ces tensions relationnelles, mais aussi attirée par les dimensions lacunaires du monde perçu. Ces «lacunes » peuvent être de deux sortes : (1) l'inachèvement de telle ou telle détermination, que la perception tend à compléter dans un processus d'investissement et de surdétermination (comme dans la vision du célèbre motif ou "triangle » de Gaetano Kanizsa) ; (2) mais aussi l'inépuisable de la profondeur ou des horizons, l'indéfini de l'indétermination. Ces dimensions lacunaires mettent le sujet percevant lui-même en tension (et en intention), le sollicitent et le configurent (voir « en » profondeur pour voir «la » profondeur), l'invitent à s'abandonner, l'obligent à s'orienter et à désirer. À être chair.

Ces mêmes tensions relationnelles et dimensions lacunaires font que le sujet percevant tend à vivre la chose perçue elle-même comme 
chair: il la saisit dans son style propre, dans les typiques de son " comportement», comme si elle était corps mobile et corps animé en relation avec son environnement. Le «style»- on sait combien le dernier Merleau-Ponty associera fortement chair et style - se compose des traits invariants d'un système de variations. Et Merleau-Ponty de prendre pour exemple caractéristique la perception d'un cercle, autrement dit d'une figure mathématique à première vue si peu charnelle. Le cercle perçu n'est pas saisi comme une essence immobile (un ensemble de points à égale distance d'un point fixe), il est vécu comme une surprise permanente. Le cercle n'est perçu qu'en étant furtivement parcouru. Ce parcours épouse une ligne mouvante (et non des points immobiles) et éprouve à chaque instant (et non à chaque endroit) que celle-ci s'écarte (de la tangente), et s'écarte toujours de la même manière, selon le même style ${ }^{5}$.

Pour Merleau-Ponty, cette épreuve de l'écart ne serait pas un cas particulier mais un ingrédient de toute perception : toute perception est épreuve de variations et repérage de leurs invariants, toute perception est épreuve (ou surprise) de la surprise, jusque dans sa dimension de reprise. La perception n'opère pas selon le modèle intellectualiste de l'intuitus; elle n'accède pas instantanément à une essence, mais prend du temps et engage la motricité. Percevoir un écart implique de le simuler, selon un parcours sensori-moteur qui mime son style : la perception navigue dans les passages qui vont de l'un à l'autre des termes de l'écart, et ainsi les relie déjà, voit en profondeur les incompossibles, porte à la fois l'ancien et le nouveau, tient ensemble ce qui est écarté. Ce faisant, la perception est déjà engagement et expression, et elle est déjà un début de reprise de la surprise. L'écart perçu, en tant que perçu, est déjà en partie contenu, la déstabilisation en partie stabilisée, la nouveauté en voie d'être intégrée, la désorientation commence à laisser place à une nouvelle orientation.

Toute chose est donc perçue par et dans notre ouverture à ses possibles lacunes comme à celles de son environnement (ouverture à l'inachèvement de ses déterminations et à l'indétermination qui l'entoure), mais aussi par et dans notre ouverture aux possibles variations de son rapport au fond et aux autres choses, et dans l'épreuve des invariants de celles-ci. Cet ensemble est loin d'être étranger à notre vécu de la surprise, tel que nous l'avons déjà dégagé. Nous sommes à... l'écart et nous mettons la chose à l'écart (de ses écarts). C'est ainsi,

${ }^{5}$ Cf. M. Merleau-Ponty, Le monde sensible et le monde de l'expression, cours au Collège de France (1953), Genève, MetisPresses, 2011, p. 49-50, 56-57. 
et seulement ainsi, que cristallise l'identité perceptive de la chose, qui n'est donc pas préalable à l'écart ${ }^{6}$. Percevoir par et dans l'épreuve d'un écart et ainsi (re)connaître la chose perçue, c'est percevoir et (re)connaître non pas malgré la surprise, mais au cœur de la surprise.

\section{Interroger}

Merleau-Ponty ne s'arrête pas à cette description phénoménologique, à dominante gestaltiste ou structurale, de la perception. Il la complète par une coloration plus animiste, qui entend assumer plus avant les aspects relationnels de la vie perceptive. L'être perçu est perçu comme une chair, traversée et animée des tensions relationnelles déjà évoquées. Le sujet percevant projette ainsi sur lui une animation - et même une animalité - qu'il a en propre, allant jusqu'à entretenir avec l'être perçu ce que Merleau-Ponty aime nommer « un véritable commerce »: une relation plus ou moins engageante et réciproque. À travers et au-delà de la perception, il nous faut envisager en quoi la surprise elle-même implique, et suppose, une forme de relation; en retour, la surprise est susceptible de vitaliser et approfondir la relation au sein de laquelle elle surgit.

a) Foi perceptive et interrogative

La phénoménologie de Merleau-Ponty souligne combien il n’y a jamais de relation neutre au monde ou à autrui. Car il n'y a pas de relation sans un minimum d'adhésion confiante, avec ce que celle-ci comporte d'endurance de l'indétermination. Mais il n'y a pas non plus de relation sans interrogation, avec ce que celle-ci comporte de mise à l'épreuve des déterminations, une épreuve qui engage le corps. Ces deux dynamiques implicantes, complémentaires, sont à l'œuvre dès la vie perceptive. Elles peuvent sembler incompatibles, l'interrogation paraissant impliquer un recul invalidant l'adhésion de la foi. Pourtant Merleau-Ponty tend à les conjoindre de diverses manières. Notamment dès sa description, que nous venons d'évoquer, de la perception comme épreuve d'un écart, et plus encore dans son analyse tardive d'une foi perceptive et interrogative (qu'il nomme parfois aussi foi naturelle ou foi animale).

\footnotetext{
${ }^{6}$ L'identité des termes est saisie dans la tension de leur écart, et simultanément à celle-ci. La différence n'apparaît pas sur fond d'une identité préalable, contrairement à la rétrospection d'une intelligence aristotélicienne qui assure chaque être, d'abord par genre puis par différence spécifique, dans une spatialité projective le stabilisant et le protégeant de la surprise des accidents.
} 
La foi est cette modalité vitale de la relation qui parie sur celle-ci et endure son indétermination, tout en s'appuyant sur des déterminations et en passant outre à leur insuffisance. Tout en éprouvant ce que nous savons et ressentons déjà, la foi va au-delà de ces affects et représentations qui ne font pas et ne feront jamais preuve, qui ne sauraient résorber la part d'invisibilité et d'imprévisibilité de la relation (ce en quoi elle nous échappe toujours en partie, dans la mesure où elle n'est pas garantie par une harmonie préétablie ou un système explicatif). Pour Merleau-Ponty, c'est là une attitude fondamentale de la chair, largement engagée dès la vie perceptive. Loin d'être purement passive et loin des illusions d'une adhésion aveugle et fusionnelle, la foi perceptive passe par la mise à l'épreuve de ce en quoi elle se confie, une épreuve interrogative. L'interrogation, de son côté, ne peut s'exercer dans le recul total d'un doute généralisé sans s'illusionner gravement sur ce qu'elle est, car elle est toujours déjà précédée par notre fréquentation de l'être, soutenue par nos «attaches ontologiques $»^{7}$. La foi perceptive et interrogative qui soutient notre étonnement, qui porte toutes nos questions, vit de cette surprise fondamentale que notre chair éprouve à être toujours déjà prise par l'être, un être qui la précède et qui, le premier, $1^{\prime}$ interroge ${ }^{8}$. Dès la moindre perception, la chair est ainsi appelée à vivre la surprise de l'être.

L'ontologie de Merleau-Ponty, sans le formuler comme tel, est l'esquisse d'un être foncièrement surprenant. Comme si l'être était fondamentalement éprouvant, et en retour fait pour être éprouvé et pour être mis à l'épreuve, pour être touché et pour nous toucher -

\footnotetext{
${ }^{7}$ Cf. E. de Saint Aubert, "La chair ouverte à la portance de l'être ", in Alter, n²3, "Anthropologies philosophiques », Dijon, Alter, 2015, notamment p. 174-177.

${ }^{8}$ Comme tant d'autres philosophes, Merleau-Ponty revient à cette racine vitale de l'intelligence qu'est l'étonnement, dans une préséance de la surprise sur notre prise, de l'étonnement sur la compréhension. Mais il n'oublie pas que la surprise, ce faisant, est une forme de prise du monde sur nous. Le monde nous prend et nous prenant nous surprend; il nous touche et c'est ainsi qu'il peut nous étonner. Nos conceptions parfois trop théorétiques de l'étonnement philosophique sont paradoxalement encombrées d'une sorte de Noli me tangere adressé au monde : je ne te touche pas, je m'étonne d'abord, mais secrètement je m'étonne pour ne pas toucher et plus encore ne pas être touché, évitant ainsi de véritablement m'étonner. La surprise sans prise, l'étonnement sans contact relèvent de pensées de survol qui défigurent l'expérience humaine et handicapent la métaphysique par leur refoulement ou refus de la promiscuité de l'être - laquelle enveloppe pourtant une dimension fondamentale de sa possible portance. Merleau-Ponty a toujours été sensible à la structure pré-autistique d'une certaine conscience philosophique, qui prend «pour type et idéal de la connaissance humaine notre contemplation des objets inanimés, des choses indifférentes, et qui ne nous touchent pas » (« Être et Avoir » (1936), in Parcours 1935-1951, Lagrasse, Verdier, 1997, p. 35, Merleau-Ponty souligne). Ses derniers écrits, fidèles à une poétique du monde charnel héritière du surréalisme et de Claudel, soulignent que les choses nous regardent, que le monde nous touche, et que nous avons toujours déjà fréquenté l'être.
} 
dans toute l'amplitude de sens de ce "toucher», jusque dans $l^{\prime}$ « affecter » le plus radical. C'est là l'une des orientations majeures de la poétique merleau-pontienne de $l^{\prime}$ «empiétement». Cette ontologie contraste ainsi fortement avec les métaphysiques de la substance qui, peu ou prou, mettent l'être à l'abri de la surprise... pour mieux nous mettre nous-mêmes à l'abri de la surprise de l'être. On peut en effet suspecter toute métaphysique de la substance, sinon toute métaphysique tout court, de garantir une fois pour toutes la solidité de l'être en nous affranchissant de la double épreuve de l'être par notre chair et de notre chair par l'être. Pour nous éviter d'entrer avec l'être dans une véritable relation, trop brûlante et engageante. La posture thétique des métaphysiques jouerait ainsi comme une croyance qui nous dispense de la foi, qui nous évite la double aventure de la foi et de l'interrogation - celles que nous portons sur l'être et celles que l'être exerce sur nous -, et qui, au fond, nous dispense de percevoir et d'être perçus.

b) Éprouver et désirer

Endurance de l'indétermination et épreuve des déterminations : ces deux conditions d'une relation vivante repuisée dans ses fondements perceptifs confirment combien notre ouverture au monde et à autrui, jusque dans ses dimensions passives, est toujours plus active qu'on pourrait le croire; combien elle implique de donner, et pas seulement de recevoir. Quels sont le sens et l'extension de ce «donner»? La mise à l'épreuve corporelle du monde engage au dehors ce corps que nous sommes, engage son expression. Par ailleurs, la foi implique toujours une forme d'abandon relatif (ou total) de soi à... lequel passe par une relative (ou totale) déposition de soi dans l'être auquel on donne foi et sur lequel on s'appuie : la foi implique une forme de don et d'abandon de notre être ${ }^{9}$. En somme, toute relation vivante suppose une implication de soi et un engagement au dehors, sans lesquels notre rapport au monde et à autrui ne constitue jamais une véritable expérience. Le vécu de la surprise est lui-même marqué par cette complexion dynamique de la relation comme de l'expérience, peut-être à titre exemplaire. La surprise est une expérience, et met en valeur une dimension fondamentale de toute expérience : éprouver, au double sens passif-actif de

\footnotetext{
${ }^{9}$ On peut le voir, en creux, dans la déstabilisation provoquée en nous par une trahison de la confiance, déstabilisation qui peut aller jusqu'à un véritable vertige identitaire: preuve que le «pari » de la foi n'est pas un simple jeu que l'on gagne ou que l'on perd, mais implique une déposition de notre être, au risque de le perdre. À travers et au-delà de ses dimensions psychologiques cognitives et affectives (parier sur la base de ce que nous savons et ressentons), la foi a une véritable dimension existentielle.
} 
ressentir et de mettre à l'épreuve. La surprise est ingrédiente de toute expérience, en tant qu'épreuve d'une nouveauté qui nous surprend et nous met à l'épreuve, que nous éprouvons et mettons à l'épreuve, que nous accueillons et interrogeons.

Ce faisant, se pose à nouveau la question de ce que nous allons intégrer de cette nouveauté. Car notre activité au sein de la passivité de la surprise ne s'arrête pas à cette exposition (de soi) et mise à l'épreuve (de l'autre) : l'ouverture à l'indétermination et l'épreuve des déterminations, ainsi conjointes dans un même geste, sont prises dans un processus d'incorporation. Or, celui-ci ne va à son terme qu'en passant du [se laisser prendre par... en s'abandonnant à...] à un [prendre (une partie) en soi, assimiler et rejeter], qu'en redoublant celui-là par celui-ci - pas forcément selon deux phases distinctes et successives, car ces deux attitudes peuvent alterner à une fréquence d'oscillation telle qu'elles paraissent simultanées. Se laisser envelopper, mais aussi envelopper ; pénétrer et se laisser pénétrer, jusqu'à introjecter, ce qui ne se fait pas sans rejet. Où nous retrouvons le double mouvement de la chair déjà évoqué - une topologie dynamique, qui tisse toute relation véritable de pénétration et d'enveloppement mutuels. Et où nous retrouvons, à travers et au-delà du geste de la foi, celui du désir.

D'une certaine manière, on ne peut pas accueillir la nouveauté et l'intégrer sans un minimum la désirer. Bien entendu, une telle affirmation implique une conception du désir qui ne l'assimile pas à l'envie, et l'enracine en deçà de toute possessivité. Qui ne l'assimile pas non plus à la pulsion, en son acception freudienne orthodoxe. Poussée irrépressible en vue de supprimer ou réguler un état de tension, la pulsion, telle que Freud la décrit avec conséquence, est conservatrice et régressive, force du retour au même, donc relativement intolérante de l'imprévu, de l'inconnu, de la nouveauté. L'envie, de son côté, est déterminée, et si la nouveauté peut contribuer à son appétit, elle n'en est pas l'objet. Ni la pulsion ni l'envie n'ont leur terrain de jeu dans l'endurance de l'indétermination, et par conséquent ni l'une ni l'autre ne suffisent à l'endurance de la surprise. Le désir, qui se joue dans l'être et pas seulement dans l'avoir (contrairement à l'envie), qui est plus structurel qu'énergétique (contrairement à la pulsion), est endurance de l'indétermination et appétit de la nouveauté.

Mais le désir n'en reste pas à l'ouverture, à l'endurance et à l'appétit de l'indétermination. Il participe, sinon préside à la mise à l'épreuve interrogative des déterminations, en vue de l'incorporation de certaines d'entre elles. Fondé dans l'être, et porté par l'être, il 
chemine aussi dans l'avoir. Inextricablement lié à l'intelligence, aussi bien dans son ouverture à l'indétermination que dans son épreuve des déterminations, il préside à l'ensemble du processus d'incorporation. Or, celui-ci se joue dans une sorte de course d'orientation, marquée par une tension continue faite de jugements et de choix. On s'oriente en se repérant et en choisissant un cap que l'on va tenir, en fixant un horizon par nature non parfaitement déterminé et en s'appuyant sur certaines déterminations tout en négligeant toutes les autres. L'incorporation exige de trier ce qui s'offre à la perception pour négliger ou rejeter une partie des déterminations (imperception), en retenir d'autres et s'en saisir tout en les complétant : les faire chair et en faire notre chair.

Après la dé-prise et la déstabilisation de la surprise, voici le temps de la re-prise ; après la perte et le vide, voici le temps du gain ; après avoir lâché et avoir plongé, voici le temps de nager ou de voler, dans la portance de l'être surprenant. Mais encore une fois, l'énoncé d'un "après » trouve surtout sa pertinence pour la clarté d'exposition: en réalité, au sein du mouvement vivant, au cœur de notre relation à la surprise, ces trois «temps » forment souvent un seul et même geste de notre chair, un seul et même événement de notre être-au-monde ou de notre être-à-autrui, tout entier traversé de foi, d'interrogation et de désir.

\section{Résistances}

Les précédentes analyses ont déjà commencé à éclairer nos résistances à la surprise. Résistances de divers types, parfois étonnamment fortes, certaines particulièrement actuelles. Elles montrent un nouveau visage de notre sourde activité face à la surprise - mais cette fois une activité qui, loin d'épauler la passivité nécessaire pour être surpris, tend à l'empêcher, afin d'échapper à la surprise. On pourrait rassembler ces moyens de résister et échapper à la surprise sous trois grandes catégories : les objets sans surprise, les croyances sans surprise, les relations sans surprise. Ces dernières sont en réalité premières : les objets anti-transitionnels et les croyances sans foi sont au service des relations sans surprise, sinon d'une fuite de la relation.

a) Ni dehors ni dedans

Dans certaines sociétés, l'être humain semble aujourd'hui avoir dramatiquement vieilli, parvenant difficilement à s'ouvrir à la surprise, plus encore à l'endurer. Serait-ce parce qu'il n'y a rien de 
nouveau sous ce qui reste du soleil, parce que nous sommes trop habitués à trop de choses, prisonniers du sempiternel retour d'une marée de déterminations chaotiques qui finit par ressembler vaguement à un même? Nous n'avons plus le temps d'être surpris : à peine une information est-elle arrivée qu'une autre suit et la remplace. Cette logique horizontale de substitution, effrénée et indéfinie, cultive une temporalité pulsionnelle et nous éloigne du temps du désir ; elle nous enchaîne à la succession d'une kyrielle de micro-problèmes, aux antipodes de la logique verticale d'approfondissement du mystère. Nous n'avons plus l'espace d'être surpris : nous sommes encombrés de tout un attirail d'objets qui nous bouchent l'horizon, une quincaillerie de visibles qui nous cachent l'invisible. Des objets transitionnels à foison qui ne font plus transition, tout un petit merdier que l'on transporte avec soi - au cas où, on ne sait jamais. En cas de surprise. Car elle peut toujours arriver... et il faut surtout y parer, par avance, et de tous les côtés. On s'attend à être surpris, mais on n'attend plus la surprise : on s'en préserve par avance, armés de fétiches.

Ce tableau est en réalité moins celui d'un être vieux et blasé que d'un individu saturé, et fatigué. Saturé de déterminations qui ne lui laissent plus l'espace et le temps de s'ouvrir à l'indétermination, et fatigué de tenter de tenir ensemble une multiplicité ingérable de choses entre lesquelles il sautille jusqu'à s'y noyer. Il ne parvient plus à construire - et à se construire - avec ces choses qui ne tiennent plus ensemble, ne font plus corps, et ainsi ne lui donnent plus la portance dont il a besoin ${ }^{10}$. Or, les choses ne tiennent ensemble que dans la profondeur d'une indétermination qui les soutient et sur laquelle elles ouvrent. Sans ce fond qui est fonds, nous ne pouvons plus les percevoir. Il nous faut décidément réapprendre à voir, entrer dans "le silence de celui qui perçoit ${ }^{11}$, jeûner de déterminations et réapprivoiser l'horizon. Pour qu'à nouveau la goutte d'eau qui tombe sur la peau de notre enveloppe psychique ne parvienne pas sur une surface déjà brouillée par une averse continue d'affects et de représentations, puisse initier et imprimer son propre train d'ondes, soit ainsi reçue par et dans sa propre impression.

Mais nous ne faisons pas que subir cette situation de saturation: nous participons à la créer et à l'entretenir. Nous multiplions les moyens de nous protéger de l'indétermination et de nous préserver

\footnotetext{
${ }^{10}$ Cf. E. de Saint Aubert, «Introduction à la notion de portance », in Archives de philosophie, tome 79, $\mathrm{n}^{\circ} 2$, avril-juin 2016, p. 317-343.

${ }^{11}$ Merleau-Ponty, notes de préparation inédites du Visible et l'invisible, B.N.F., volume VII, f. $[136] v(4)$.
} 
de la surprise - certains moyens sont largement répandus, voire mondialisés, d'autres sont davantage liés à notre idiosyncrasie ou à notre métier. Ils décrivent toutes les manières de se protéger du vrai dehors... et du vrai dedans - de se préserver de celui-là pour se protéger de celui-ci. Car si la surprise semble d'abord venir du dehors et $\mathrm{s}^{\prime}$ annoncer même comme un pur dehors, inconnu ou étranger, tel un adversaire qui nous convoque et nous provoque, elle peut aussi venir du familier et de l'habituel : d'un dedans, si proche et si connu que cette promiscuité induit une sourde ignorance, parfois massive. Mais le plus étonnant est la secrète conjonction des deux, car le dehors est souvent apte à exprimer le dedans, l'être peut nous renvoyer des reflets de nous-mêmes. La surprise du dehors et celle du dedans, la surprise de l'être et la surprise de l'inconscient se conjuguent alors et se renforcent dans la surprise du miroir, où le dehors surprend notre dedans, où nous sommes invités à saisir un dehors d'autant plus saisissant qu'il s'est saisi de nous.

Dans notre résistance à la surprise, nous nous employons à laisser le dehors au dehors et le dedans au dedans, pourvu qu'ils ne bougent pas et ne nous bougent pas, pourvu surtout qu'ils ne communiquent pas et s'échangent encore moins, car alors nous serions traversés, et exposés. Nos moyens de résistance déclinent toutes les manières de nous protéger du renvoi expressif entre le dehors et le dedans, de leur réversibilité dans l'incorporation, de leur communion dans le désir. De nous soustraire à la surprise de l'être et à celle de l'inconscient ${ }^{12}-$ le tout, bien souvent, dans un idéal de pleine conscience, avec l'illusion que celle-ci nous ouvre pleinement au monde, à autrui et à nousmême, alors que sa lucidité aveugle joue d'interface préservatrice entre le dehors et le dedans ${ }^{13}$. La surprise à laquelle nous sommes

\footnotetext{
12 À propos de cette conjonction entre l'être et l'inconscient, cf. E. de Saint Aubert, « La conception merleau-pontienne de l'inconscient dans les manuscrits tardifs ", in Approches phénoménologiques de l'inconscient, M. Gyemant et D. Popa (éd.), Hildesheim/Zürich/New York, Georg Olms Verlag, 2015, p. 187-209.

${ }^{13}$ L'utopie de la pleine conscience est souvent secrètement motivée par une conduite de sauvegarde et de fuite, déniant et tentant d'exorciser le non-sens qui nous hante, l'intolérable invisible qui nous taraude. Elle est l'exemple type d'une posture qui se fait passer pour une attitude spirituelle pure alors qu'elle est encombrée de motivations psychologiques verrouillant notre ouverture au monde. Il n'en demeure pas moins vrai que la conscience participe à nous ouvrir au monde, à autrui et à nous-même, et que cette participation est capitale - la question n'est pas là. Mais il reste et restera toujours une certaine contrariété entre conscience et ouverture, parce que la conscience, malgré ce qu'on aimerait croire, n'est pas véritablement extatique. Merleau-Ponty, marqué par son travail ininterrompu de la condition perceptive de notre être-au-monde, apporte à ce sujet une réelle contribution, qui culmine dans sa conception tardive de l'inconscient. Cf. à nouveau E. de Saint Aubert, «La conception merleau-pontienne de l'inconscient dans les manuscrits tardifs ", art. cit.
} 
susceptibles de résister le plus n'est autre que nous-même. Nousmême, comme un autre avec lequel nous ne voulons pas entrer en relation, ou du moins changer de relation.

b) Fétichisme et objectivisme

Nos résistances à la surprise s'inscrivent largement dans nos tentatives de maîtriser la relation avec autrui, en nous protégeant de l'indétermination de celle-ci, en particulier en voulant thésauriser et contrôler la présence d'autrui. C'est la grande aventure de la représentation, née au cœur de l'hallucination de la présence de la mère en son absence, parce que cette présence est relative et incontrôlable, et qu'il faut à tout prix pouvoir se la donner à soi-même et en jouir à volonté. Si cette aventure inaugure bientôt celle de l'objet transitionnel, elle ouvre aussi, de manière indissociable, les mésaventures du fétichisme.

Le fétichiste ne paraît pas résister directement à la surprise : il ne $\mathrm{s}^{\prime}$ agit pas tant pour lui de se préserver de l'invisible et de l'imprévisible que de préserver le visible, de conserver et répéter le déjà vu et connu. Pourtant ceci revient à cela, et ceci est en partie en vue de cela. Le fétichiste est dans l'ambition de tout voir, de tout rendre visible et prévisible. Ce qui implique une parfaite distribution de l'espace, où chaque chose s'étend, à son emplacement unique, sous son œil de cyclope. Ce qui implique une pétrification du temps, pour immobiliser chaque trait du réel et empêcher tout changement. Cette totalisation et cette instantanéité visent à pouvoir jouir pleinement de la présence, laquelle est réduite à une pure passivité, 1 "« autre » ayant subi une réduction de toute action et de toute expression (de toute liberté, pourrait-on ajouter), ainsi qu'une réduction de tout ressort intérieur et caché (de tout désir). Ainsi réduite à une trame d'inertie, pétrifiée en chose passive, parfaitement repérée, disponible et manipulable, la présence congelée est perdue comme telle. Et la relation, déterminée, n'a plus d'avenir.

On aura ici reconnu certains éléments de l'approche sartrienne de la chair, description dont la chair merleau-pontienne entend précisément prendre le contrepied ${ }^{14}$. Merleau-Ponty ne parle pas du fétichisme, mais sa critique de Sartre et plus largement sa lecture quasi psychanalytique de l'objectivisme le décrivent à sa manière, et contribuent à éclairer nos résistances à la surprise. L'objet idéal, nous l'avons vu (supra, 3-a), est par construction affranchi de l'inaccessible

\footnotetext{
${ }^{14}$ Cf. E. de Saint Aubert, Du lien des êtres aux éléments de l'être, Paris, Vrin, 2004, notamment section A, chap. III.
} 
et de l'indétermination; mais tout se passe comme s'il était en partie construit dans ce but. L'ontologie de l'objet est soutenue par des conduites de fuite de l'indétermination et de sauvegarde de la détermination, et se replie dans les illusions de la conscience - illusions de savoir, d'avoir et de pouvoir -, celles d'un observateur désengagé. Ce faisant, elle dénie la condition humaine, aux prises avec les surprises de l'indétermination, et referme les conditions d'exercice de la foi perceptive, de l'espérance et du désir, qui ne sauraient se déployer dans la pure détermination. La Nature ou le monde $d u$ silence évoque cette "sagesse désespérée », où "tout est décidé $\mathrm{d}^{\prime}$ avance » et où "il $\mathrm{n}^{\prime} \mathrm{y}$ a plus rien à chercher ${ }^{15}$, où rien ne peut plus nous surprendre, ni nous étonner.

Tel que Merleau-Ponty le décrit, l'objet n'est pas construit comme accessible et déterminé par la seule résorption des horizons et profondeur extérieurs, il est aussi dépourvu d'horizons et de profondeur intérieurs : surfacique, lisse et impénétrable, il n'a plus cette épaisseur et ce relief qui pourraient nous surprendre et nous agresser. Il n'a plus cette chair de la chose qui peut nous investir et nous hanter. L'objet est ainsi mis à l'abri de tout débordement de lui-même par le dehors comme par le dedans, à l'abri de la surprise de l'être comme de celle de l'inconscient. Et, plus encore, à l'abri de leur rencontre dans la chair : il est abstrait de tout échange du dehors et du dedans, ce qui revient à le mettre (à nous mettre) à l'écart de toute relation désirante avec nous (avec lui). L'objet n'a plus rien de substantiel à échanger avec nous, et ne nous interroge plus. Il ne s'expose pas à cette dimension fondamentale de l'expérience qu'est la mise à l'épreuve de la consistance de l'être dans un corps à corps; nous n'avons pas à exposer et prêter notre corps pour le rencontrer. L'objet habite la pureté adamantine d'un espace hors chair, abstrait de tout rapport charnel. Si bien que sa parfaite accessibilité l'enferme paradoxalement dans une inaccessibilité totale. Seule condition pour qu'il soit, radicalement, sans surprise.

L'ontologie de Merleau-Ponty, notamment marquée par sa conception de la peinture moderne, s'emploie à faire voler en éclat cette ontologie de l'objet, construite hors adversité, hors désir et hors chair. Et ce, depuis certains manuscrits de la fin des années quarante, qui offrent une remarquable anticipation de L'CEil et l'Esprit. Face aux objets pétrifiés et stupides, construits à la mesure de nos prises pour nous mettre à l'abri de la surprise des ultra-choses, Merleau-Ponty introduit l'objet-trouvaille du surréalisme de Breton, cristallisation du

${ }^{15}$ M. Merleau-Ponty, La Nature ou le monde du silence, B.N.F., vol. VI, f. [50](3). 
désir, merveilleux précipité du visage humain. Cet objet déborde et nous déborde par le dehors comme par le dedans; il nous parle de nous-même et nous introduit au mystère. Car tout objet est susceptible de (re)devenir transitionnel, et de nous rouvrir ainsi à la surprise de l'être. Tout se passe comme si les choses elles-mêmes résistaient à notre résistance, et Merleau-Ponty en vient à évoquer ces objets qui « saignent » et nous " agressent», motif audacieux inauguré dans la conférence de 1948 sur L'homme et l'objet ${ }^{16}$. Les objets saignent, autrement dit crient qu'ils ne sont pas objets, et revendiquent la pulpe des ultra-choses; eux-mêmes ont une chair, s'expriment et nous expriment, et "nous arrachent à nous-mêmes ». C'est ainsi qu'ils nous surprennent. Ils nous renvoient à notre condition charnelle, celle $\mathrm{d}^{\prime}$ " une humanité sans stabilité, libre, inventive et sans cesse menacée », qui a « le sens de l'étonnement et du mystère ${ }^{17}$.

\footnotetext{
${ }^{16}$ Conférence inédite résumée par J.-L. Dumas, «Les Conférences », in La Nef, 5e année, $\mathrm{n}^{\circ} 45$, août 1948, p. 150-151.

${ }_{17} \mathrm{Ibid}$. Pour une analyse de ces textes et un développement de ces idées, cf. E. de Saint Aubert, Du lien des êtres aux éléments de l'être, op. cit., section B.
} 